\title{
Phase properties of light propagating in a Kerr medium: Stokes parameters versus Pegg-Barnett predictions
}

\author{
A. Luis, ${ }^{*}$ L. L. Sánchez-Soto, ${ }^{*}$ and R. Tanaś \\ Nonlinear Optics Division, Institute of Physics, Adam Mickiewicz University, 60-780 Poznań, Poland
}

(Received 9 June 1994)

\begin{abstract}
The quantum theory of light propagation in a Kerr medium is applied to describe changes in the polarization state of light. A number of purely quantum effects arising during the propagation are analyzed. Stokes operators and Pegg-Barnett formalism are used to describe the evolution of the polarization ellipse. The predictions of these approaches are compared, showing some striking differences in the quantum regime of small photon numbers.
\end{abstract}

PACS number(s): 42.50.Dv, 03.65.Bz, 42.50.Ar, 42.50.Lc

\section{INTRODUCTION}

Recently the nonlinear interaction of a radiation field with a Kerr medium has attracted a great deal of attention. It is well known that when strong elliptically polarized light propagates through an isotropic nonlinear medium, the medium becomes birefringent, which results in the self-induced rotation of the polarization ellipse [1]. This effect can be understood in terms of the classical behavior of field amplitudes; however, if quantum fluctuations are taken into account some new effects can occur, such as photon antibunching [2,3], squeezing [4], or the generation of discrete superpositions of coherent states [5-7]. In addition, a Kerr medium is a good candidate to perform quantum nondemolition measurements of the photon number [8]. So there is a growing interest in revealing those aspects of nonlinear propagation which are directly related to the quantum properties of the field.

The purpose of this paper is to describe the effects associated with the propagation of elliptically polarized light through a lossless Kerr medium. To this end, a two-mode description of the field is needed, although for circularly polarized light the problem can be reduced to a one-mode problem [9-11], which is equivalent to the anharmonic oscillator model so widely studied [12].

To discuss the phase properties of the field, we first need a convenient description of the phase at the quantum level. Unfortunately, until now there is not a completely accepted phase operator and a number of different solutions have been proposed [13-15]. In this context, the propagation through a Kerr medium can be quite interesting since, even in the classical domain, it shows a nontrivial evolution of the phase, while the intensity corresponding to the circularly polarized amplitudes remains a constant of motion. Thus the propagation can be ascribed just to phase changes.

*Permanent address: Departamento de Óptica, Facultad de Ciencias Físicas, Universidad Complutense, 28040 Madrid, Spain.
The polarization state of the field can be described by the Stokes parameters, which are the expectation values of the corresponding Stokes operators when the quantum description of the field is used. These parameters are related to the phase difference of the two circularly orthogonal polarizations. Since they are directly measurable quantities, they can be used to define an operational way of measuring the phase difference. Moreover, a polar decomposition of these Stokes operators has been used recently to define a phase-difference operator $[16,17]$.

On the other hand, the Hermitian phase formalism introduced by Pegg and Barnett [18], which has received a lot of attention in recent years, allows a direct calculation of the mean values and variances of the phase for the two modes of the polarized light as well as their correlations. Its consequences for the problem at hand have been fully analyzed by Gantsog and Tanaś [19].

The main purpose of this work is to compare these two ways of evaluating the phase-difference changes during propagation. We will show that their structure is quite similar but, while the Pegg-Barnett approach deals with a continuous phase difference, the other one takes values only on a numerable set, which brings about some differences for fields with a small number of photons.

\section{DYNAMICAL EVOLUTION OF THE FIELD PROPAGATING IN A KERR MEDIUM}

\section{A. Summary of the classical results}

The induced polarization in a Kerr medium can be written in the form [20]

$$
\mathbf{P}=\chi \mathbf{E}+A\left(\mathbf{E} \cdot \mathbf{E}^{*}\right) \mathbf{E}+B(\mathbf{E} \cdot \mathbf{E}) \mathbf{E}^{*},
$$

where $\chi$ is the linear susceptibility of the medium and $A$ and $B$ constants characterizing the nonlinearity.

In order to obtain the evolution of the field amplitudes of a plane wave of frequency $\omega$, we consider onedimensional propagation and thus express the electric field as 


$$
\mathbf{E}=\left(\varepsilon_{x} \mathbf{e}_{x}+\varepsilon_{y} \mathbf{e}_{y}\right) e^{i(k z-\omega t)},
$$

where $k=(\omega / c) \sqrt{1+4 \pi \chi}$, and $\mathbf{e}_{x}$ and $\mathbf{e}_{y}$ are the unit vectors of a Cartesian basis. Usually it is more convenient to work in a circular basis for the polarization of the field

$$
\mathbf{e}_{ \pm}=\frac{\mathbf{e}_{x} \pm i \mathbf{e}_{y}}{\sqrt{2}}
$$

Accordingly, the amplitudes of these circularly polarized modes can be written as

$$
\varepsilon_{ \pm}=\frac{\varepsilon_{x} \mp i \varepsilon_{y}}{\sqrt{2}}
$$

and satisfy

$$
\begin{gathered}
\left|\varepsilon_{x}\right|^{2}+\left|\varepsilon_{y}\right|^{2}=\left|\varepsilon_{+}\right|^{2}+\left|\varepsilon_{-}\right|^{2}, \\
2 \varepsilon_{+} \varepsilon_{-}=\varepsilon_{x}^{2}+\varepsilon_{y}^{2} .
\end{gathered}
$$

It is easy to check that the intensities of both circular components $\left|\varepsilon_{ \pm}\right|^{2}$ are constants of the motion and then the Maxwell equations in terms of these amplitudes can easily be solved in the slowly varying approximation, obtaining

$$
\begin{aligned}
\varepsilon_{ \pm}(z)= & \exp \left\{i \frac { 2 \pi \omega ^ { 2 } } { k c ^ { 2 } } \left\{A\left[\left|\varepsilon_{+}(0)\right|^{2}+\left|\varepsilon_{-}(0)\right|^{2}\right]\right.\right. \\
& \left.\left.+2 B\left|\varepsilon_{\mp}(0)\right|^{2}\right\} z\right\} \varepsilon_{ \pm}(0)
\end{aligned}
$$

The effect of the nonlinear term appears as an intensity-dependent phase of the field (self-phase modulation or intensity-dependent refractive index). Thus the polarization changes due to the nonlinear interaction, which results in the self-induced rotation of the polarization ellipse. This change can easily be accounted for with the use of the Stokes parameters, which in terms of the circular amplitudes can be expressed as [21]

$$
\begin{aligned}
& s_{0}=\left|\varepsilon_{+}\right|^{2}+\left|\varepsilon_{-}\right|^{2}=|\varepsilon|^{2}, \\
& s_{1}=\varepsilon_{+}^{*} \varepsilon_{-}+\varepsilon_{-}^{*} \varepsilon_{+}=|\varepsilon|^{2} \cos 2 \eta \cos \phi, \\
& s_{2}=i\left(\varepsilon_{-}^{*} \varepsilon_{+}-\varepsilon_{+}^{*} \varepsilon_{-}\right)=-|\varepsilon|^{2} \cos 2 \eta \sin \phi, \\
& s_{3}=\left|\varepsilon_{+}\right|^{2}-\left|\varepsilon_{-}\right|^{2}=|\varepsilon|^{2} \sin 2 \eta,
\end{aligned}
$$

where $|\varepsilon|^{2}$ is the total intensity, $\phi$ is the phase difference between the $\varepsilon_{ \pm}$amplitudes and defines the azimuth of the polarization ellipse, and $\eta$ describes the ellipticity parameter with the sign defining its handedness. These Stokes parameters are the more convenient characterization of the state of polarization of the field. In particular, the change in the phase difference can be obtained as

$$
\phi=-\arctan \left(\frac{s_{2}}{s_{1}}\right) .
$$

Equation (2.6) gives then for the phase difference at any point $z$

$$
\phi(z)=\phi(0)-\frac{2 \pi \omega^{2}}{k c^{2}} 2 B\left[\left|\varepsilon_{+}(0)\right|^{2}-\left|\varepsilon_{-}(0)\right|^{2}\right] z .
$$

\section{B. Quantum evolution}

As we did in the classical case, we are going to consider the evolution of a two-mode monochromatic field of frequency $\omega$. The electric-field operator can be expressed as

$$
\mathbf{E}=i \sqrt{\frac{2 \pi \hbar \omega}{n^{2}(\omega) V}}\left(a_{+} \mathbf{e}_{+}+a_{-} \mathbf{e}_{-}\right) e^{i(k z-\omega t)},
$$

where $n(\omega)$ is the linear refractive index, $V$ is the quantization volume, $\mathbf{e}_{+}, \mathbf{e}_{-}$the unit vectors associated with circularly right- and left-polarized light, and $a_{+}$and $a_{-}$ the annihilation operators for the corresponding polarized mode. The evolution of the field can be described by the following effective interaction Hamiltonian:

$$
H=\frac{\hbar}{2}\left[\kappa_{1}\left(a_{+}^{\dagger^{2}} a_{+}^{2}+a_{-}^{\dagger^{2}} a_{-}^{2}\right)+2 \kappa_{2} a_{+}^{\dagger} a_{+} a_{-}^{\dagger} a_{-}\right],
$$

where

$$
\kappa_{1}=\frac{4 \pi^{2} \hbar \omega^{2}}{n^{4}(\omega) V} A, \quad \kappa_{2}=\frac{4 \pi^{2} \hbar \omega^{2}}{n^{4}(\omega) V}(A+2 B) .
$$

The evolution of the field amplitudes can be solved in terms of the unitary evolution operator $U(t)=$ $\exp (-i t H / \hbar)$

$$
a_{ \pm}(t)=U^{\dagger}(t) a_{ \pm}(0) U(t) .
$$

After replacing the time $t$ by $-\tau$ where $\tau=n(\omega) z / c$, to deal with propagation rather than a field in a cavity, we get

$$
\begin{aligned}
a_{ \pm}(\tau)= & \exp \left\{i \tau\left[\kappa_{1} a_{ \pm}^{\dagger}(0) a_{ \pm}(0)+\kappa_{2} a_{\mp}^{\dagger}(0) a_{\mp}(0)\right]\right\} \\
& \times a_{ \pm}(0) .
\end{aligned}
$$

The close correspondence can be seen between this quantum solution for the annihilation operators and the classical solution (2.6). However, as we are going to see, the expectation values of the field operators will have different properties than their classical counterparts.

\section{PHASE PROPERTIES OF THE FIELD}

\section{A. Stokes parameters}

To discuss the quantum properties of the field, it is convenient to define the Hermitian Stokes operators as $[22]$

$$
\begin{gathered}
S_{0}=\left(a_{+}^{\dagger} a_{+}+a_{-}^{\dagger} a_{-}\right), \\
S_{1}=\left(a_{+}^{\dagger} a_{-}+a_{-}^{\dagger} a_{+}\right), \\
S_{2}=i\left(a_{-}^{\dagger} a_{+}-a_{+}^{\dagger} a_{-}\right), \\
S_{3}=\left(a_{+}^{\dagger} a_{+}-a_{-}^{\dagger} a_{-}\right),
\end{gathered}
$$




$$
\left[S_{j}, S_{k}\right]=2 i \epsilon_{j k l} S_{l}, \quad\left[S_{j}, S_{0}\right]=0
$$

The noncommutability of the Stokes operators precludes the simultaneous measurement of the physical quantities represented by them. Apart from a factor of 2 , the operators $S_{j}(j=1,2,3)$ coincide with an angular momentum, while $S_{0}$ represents the total number operator.

In order to show that the operators (3.1) are the quantum analog of the classical Stokes parameters, i.e.,

$$
s_{\mu}=\left\langle S_{\mu}\right\rangle
$$

where $\mu=0, \ldots, 3$, let us compute their mean value for a two-mode coherent state

$$
\left|\alpha_{+}, \alpha_{-}\right\rangle=e^{-\left(\left|\alpha_{+}\right|^{2}+\left|\alpha_{-}\right|^{2}\right) / 2} \sum_{n_{+}, n_{-}=0}^{\infty} \frac{\alpha_{+}^{n_{+}} \alpha_{-}^{n_{-}}}{\sqrt{n_{+} ! n_{-} !}}\left|n_{+}, n_{-}\right\rangle
$$

which has a special significance in describing the classical limit of the system, and where $\left|n_{+}, n_{-}\right\rangle$is an eigenstate of both $a_{+}^{\dagger} a_{+}$and $a_{-}^{\dagger} a_{-}$. It is easy to get [23]

$$
\begin{aligned}
s_{0} & =\left|\alpha_{+}\right|^{2}+\left|\alpha_{-}\right|^{2}=|\alpha|^{2}, \\
s_{1} & =2\left|\alpha_{+}\right|\left|\alpha_{-}\right| \cos \left(\phi_{+}-\phi_{-}\right) \\
& =|\alpha|^{2} \cos \left(\phi_{+}-\phi_{-}\right) \cos 2 \eta, \\
s_{2} & =-2\left|\alpha_{+}\right|\left|\alpha_{-}\right| \sin \left(\phi_{+}-\phi_{-}\right) \\
& =-|\alpha|^{2} \sin \left(\phi_{+}-\phi_{-}\right) \cos 2 \eta, \\
s_{3} & =\left|\alpha_{+}\right|^{2}-\left|\alpha_{-}\right|^{2}=|\alpha|^{2} \sin 2 \eta,
\end{aligned}
$$

where $\alpha_{i}=\left|\alpha_{i}\right| \exp \left(i \phi_{i}\right), \phi_{i}$ being the classical phase of the state, $|\alpha|^{2}$ is the total mean number of photons in the field, and $\eta$ defines the ellipticity. It is evident that (3.5) are exactly the Stokes parameters for two classical oscillations of amplitudes $\left|\alpha_{i}\right|$ and phases $\phi_{i}$.

One possible and natural way to characterize the phase difference in this quantum case would be through the analogous classical relation $(2.8)$ as

$$
\phi=-\arctan \left(\frac{s_{2}}{s_{1}}\right)
$$

however, we should note that this is not the mean value of any phase-difference operator. One may think also in terms of the normalized parameter $s_{1}$ as a measure of the phase-difference cosine defined as

$$
\cos \phi=\frac{s_{1}}{\sqrt{s_{1}^{2}+s_{2}^{2}}} .
$$

As discussed in detail in Ref. [23] the proper normalization is $\left[s_{1}^{2}(0)+s_{2}^{2}(0)\right]^{1 / 2}$, which leads to results close to the expectation value of the phase-difference cosine.

Next, we need to examine the dynamical evolution of the system. If the solutions (2.14) are inserted into (3.1) exact solutions are obtained for the evolution of the Stokes operators. If at $\tau=0$ the field is in a coherent state $\left|\alpha_{+}, \alpha_{-}\right\rangle$, then the field at time $\tau$ can be obtained as

$$
\begin{aligned}
U(\tau)\left|\alpha_{+}, \alpha_{-}\right\rangle= & e^{-\left(\left|\alpha_{+}\right|^{2}+\left|\alpha_{-}\right|^{2}\right) / 2} \sum_{n_{+}, n_{-}=0}^{\infty} \frac{\left(\alpha_{+}\right)^{n_{+}}\left(\alpha_{-}\right)^{n_{-}}}{\sqrt{n_{+} ! n_{-} !}} \\
& \times \exp \left(i \tau \left\{\frac { \kappa _ { 1 } } { 2 } \left[n_{+}\left(n_{+}-1\right)\right.\right.\right. \\
& \left.\left.\left.+n_{-}\left(n_{-}-1\right)\right]+\kappa_{2} n_{+} n_{-}\right\}\right)\left|n_{+}, n_{-}\right\rangle
\end{aligned}
$$

Therefore we can calculate the Stokes parameters for the resulting field. The results are

$$
\begin{aligned}
s_{0}(\tau)= & \left|\alpha_{+}\right|^{2}+\left|\alpha_{-}\right|^{2}=|\alpha|^{2}, \\
s_{1}(\tau)= & 2 \operatorname{Re}\left\{\alpha _ { + } ^ { * } \alpha _ { - } \operatorname { e x p } \left[\left(e^{i \tau\left(\kappa_{2}-\kappa_{1}\right)}-1\right)\left|\alpha_{+}\right|^{2}\right.\right. \\
& \left.\left.+\left(e^{-i \tau\left(\kappa_{2}-\kappa_{1}\right)}-1\right)\left|\alpha_{-}\right|^{2}\right]\right\} \\
s_{2}(\tau)= & 2 \operatorname{Im}\left\{\alpha _ { + } ^ { * } \alpha _ { - } \operatorname { e x p } \left[\left(e^{i \tau\left(\kappa_{2}-\kappa_{1}\right)}-1\right)\left|\alpha_{+}\right|^{2}\right.\right. \\
& \left.\left.+\left(e^{-i \tau\left(\kappa_{2}-\kappa_{1}\right)}-1\right)\left|\alpha_{-}\right|^{2}\right]\right\} \\
s_{3}(\tau)= & \left|\alpha_{+}\right|^{2}-\left|\alpha_{-}\right|^{2} .
\end{aligned}
$$

The phase difference defined by (3.6) gives in this case

$$
\phi(\tau)=\phi_{0}-\left(\left|\alpha_{+}\right|^{2}-\left|\alpha_{-}\right|^{2}\right) \sin \left[\tau\left(\kappa_{2}-\kappa_{1}\right)\right] .
$$

We can see here a first difference with the classical results. The classical solution is obtained when only the linear term in $\tau\left(\kappa_{2}-\kappa_{1}\right)$ of a series expansion of $\sin \left[\tau\left(\kappa_{2}-\right.\right.$ $\left.\kappa_{1}\right)$ ] is retained. Thus the classical limit (2.6) is recovered for small values of $\tau\left(\kappa_{2}-\kappa_{1}\right)$, but not in the limit of high intensities as could be expected.

According to (3.7), we have for the cosine of the phase difference

$$
\begin{aligned}
(\cos \phi)(\tau)= & e^{\left\{\cos \left[\tau\left(\kappa_{2}-\kappa_{1}\right)\right]-1\right\}|\alpha|^{2}} \\
& \times \cos \left\{\phi_{0}-\left(\left|\alpha_{+}\right|^{2}-\left|\alpha_{-}\right|^{2}\right) \sin \left[\tau\left(\kappa_{2}-\kappa_{1}\right)\right]\right\} .
\end{aligned}
$$

The classical solution can be recovered again in the same conditions as above. Also, this expression is not the cosine of (3.10). In general, for any operator, a function of its mean value is different from the mean value of the function. However, it should be noted that these quantities are not the quantum expectation values of a phasedifference operator, although they are defined in terms of measurable quantities (but noncommuting).

\section{B. Phase difference via Stokes operators}

The Stokes operators contain all the relevant information to characterize the polarization of the field. However, until now we have not yet characterized the phase difference itself; that is, we have not extracted a phasedifference operator from the Stokes operators.

In fact, the variable canonically conjugated to the phase-difference operator is the number difference, which is not bounded from below. So it is reasonable to expect the existence of a phase-difference operator free from the problems arising in the one-mode case where, as is well 
known, the absence of a proper phase operator in the infinite-dimensional Hilbert space is usually ascribed to the semiboundedness of the spectrum of the number operator.

If we denote $s_{ \pm}=\left(s_{1} \pm i s_{2}\right) / 2$, then the classical phase difference between the two modes is unambiguously obtained as

$$
s_{+}=e^{-i \phi} \sqrt{s_{-} s_{+}}
$$

where $\phi=\phi_{+}-\phi_{-}$. It might be supposed that there is an operator decomposition corresponding to (3.12). The quantum analog of the separation of a complex amplitude into a real part and a phase factor is a polar decomposition. So let us try to decompose the quantum Stokes operators $S_{ \pm}=\left(S_{1} \pm i S_{2}\right) / 2$ in the form

$$
S_{-}=E \sqrt{S_{+} S_{-}}, \quad S_{+}=E^{\dagger} \sqrt{S_{-} S_{+}} .
$$

When $E$ is unitary, it defines a Hermitian phasedifference operator by $E=e^{i \Phi}$. As in the onedimensional case, this polar decomposition does not completely define the exponential of phase. In our case, the matrix elements $\left\langle n_{+}, 0|E| 0, n_{-}\right\rangle$are undefined and thus $E$ cannot be uniquely determined by the unitarity requirement. We must impose then further conditions, the most adequate being commutation relations. Among them, the unique condition compatible with (3.13) and unitarity is

$$
\left[E, S_{0}\right]=0,
$$

which translates the fact that in the classical domain the form of the polarization ellipse is independent of the intensity. Therefore we shall consider the polar decomposition (3.13) together with the condition (3.14).

Since the operator $E$ commutes with the total number $a_{+}^{\dagger} a_{+}+a_{-}^{\dagger} a_{-}$, we may rather study its restriction to each subspace $\mathcal{H}_{n}$ having $n$ quanta. Calling this restriction $E^{(n)}$, Eq. (3.13) can easily be solved, obtaining the unitary operator [16]

$$
\begin{aligned}
E^{(n)}= & \sum_{m=1}^{n}|m-1, n-m+1\rangle\langle m, n-m| \\
& +e^{i(n+1) \phi_{0}^{(n)}}|n, 0\rangle\langle 0, n|,
\end{aligned}
$$

where $\phi_{0}^{(n)}$ is an arbitrary phase. Since it seems reasonable that the phase difference between two states should be independent of any phase of reference, we must impose further conditions on $E$. To this end, let us introduce the exchange operator $\Pi$, such that

$$
\Pi|n, m\rangle=|m, n\rangle .
$$

It is easy to see that $\Pi S_{-} \Pi^{\dagger}=S_{+}$and therefore we must have

$$
\Pi E \Pi^{\dagger}=E^{\dagger},
$$

which is the expected action of the exchange operator on the exponential of the phase difference, even at the classical level. Imposing now condition (3.17) on (3.15), we easily get that the allowed values of $\phi_{0}^{(n)}$ are

$$
\phi_{0}^{(n)}=\frac{\ell \pi}{n+1}
$$

$\ell$ being an integer. In each subspace $\mathcal{H}_{n}$ there are $n+1$ orthonormal states verifying that

$$
E^{(n)}\left|\phi_{r}^{(n)}\right\rangle=e^{i \phi_{r}^{(n)}}\left|\phi_{r}^{(n)}\right\rangle
$$

with $r=0, \ldots, n$. These states can be expressed in the number basis as

$$
\left|\phi_{r}^{(n)}\right\rangle=\frac{1}{\sqrt{n+1}} \sum_{n_{+}=0}^{n} e^{i n_{+} \phi_{r}^{(n)}}\left|n_{+}, n-n_{+}\right\rangle
$$

where $\phi_{r}^{(n)}=\phi_{0}^{(n)}+\frac{2 \pi r}{n+1}$.

The expression for $\stackrel{n+1}{E}$ on the whole space is

$$
E=\sum_{n=0}^{\infty} E^{(n)}=\sum_{n=0}^{\infty} \sum_{r=0}^{n}\left|\phi_{r}^{(n)}\right\rangle e^{i \phi_{r}^{(n)}}\left\langle\phi_{r}^{(n)}\right|,
$$

which is the operator recently introduced by Luis and Sánchez-Soto [16].

Since $E$ is unitary, it defines a Hermitian phasedifference operator

$$
\Phi=\sum_{n=0}^{\infty} \sum_{r=0}^{n}\left|\phi_{r}^{(n)}\right\rangle \phi_{r}^{(n)}\left\langle\phi_{r}^{(n)}\right| .
$$

In order to have the eigenvalues for $\Phi$ in the interval $(-\pi, \pi)$ (and then $\left.\Pi \Phi \Pi^{\dagger}=-\Phi\right)$ we must take

$$
\phi_{0}^{(n)}=-\frac{n \pi}{n+1} .
$$

There are some differences between this operator and other approaches. In the first place (3.21) is constructed from the start on the whole infinite-dimensional space. It is unitary and allows the definition of the phase-difference operator (3.22), and, finally, its spectrum is discrete, although in the limit of high $n$ it becomes dense, as might be expected.

With all this in mind, we can give the evolution of the phase difference for the state (3.8) as

$$
\langle\Phi(\tau)\rangle=\sum_{n=0}^{\infty} \sum_{r=0}^{n} \phi_{r}^{(n)}\left|\left\langle\phi_{r}^{(n)} \mid \psi(\tau)\right\rangle\right|^{2},
$$

and similarly for its cosine

$$
\langle\cos \Phi(\tau)\rangle=\sum_{n=0}^{\infty} \sum_{r=0}^{n} \cos \phi_{r}^{(n)}\left|\left\langle\phi_{r}^{(n)} \mid \psi(\tau)\right\rangle\right|^{2} .
$$

\section{Pegg-Barnett phase difference}

The Pegg-Barnett approach avoids the problems in the definition of a phase operator for a one-mode field by considering, as a first step, a finite-dimensional Hilbert space 
for the system. Their procedure results in a prescription of a phase probability distribution for a given state of the field in terms of the Susskind-Glogower phase states. The phase difference is obtained simply in terms of the difference of the phases for the modes involved. Then the mean value of an arbitrary function of the phase difference for a state $|\psi\rangle$ is given by

$$
\left\langle f\left(\Phi_{\mathrm{PB}}\right)\right\rangle=\int d \phi_{+} \int d \phi_{-} f\left(\phi_{+}-\phi_{-}\right) P_{\psi}\left(\phi_{+}, \phi_{-}\right),
$$

where $\phi_{+}$and $\phi_{-}$refer to the phases of individual modes and for physical states the continuous joint phase distribution function $P_{\psi}\left(\phi_{+}, \phi_{-}\right)$is obtained in terms of the Susskind-Glogower phase states

$$
\left|\phi_{+}, \phi_{-}\right\rangle=\frac{1}{2 \pi} \sum_{n_{+}, n_{-}=0}^{\infty} e^{i\left(n_{+} \phi_{+}+n_{-} \phi_{-}\right)}\left|n_{+}, n_{-}\right\rangle
$$

as $[18]$

$$
P_{\psi}\left(\phi_{+}, \phi_{-}\right)=\left|\left\langle\phi_{+}, \phi_{-} \mid \psi\right\rangle\right|^{2} .
$$

Note that integrating the distribution $P_{\psi}\left(\phi_{+}, \phi_{-}\right)$over one of the phases $\phi_{+}$or $\phi_{-}$leads to a marginal distribution for individual phases. The behavior of these marginal distributions has been fully investigated in Ref. [19].

It is worth mentioning that in this formalism the phasedifference operator is defined in a natural way as the difference of the single-mode phases. However, the $4 \pi$ eigenvalue range of the phase difference adds further subtlety to the interpretation of the joint phase distribution. When we are interested only in the difference of the two single-mode phases (and not in the individual phases), then it is more meaningful to restrict the difference to a single $2 \pi$ range, which makes it easier to interpret the results. In the Appendix we further discuss this casting procedure [24].

In this spirit, we obtain for the mean value of the phase difference

$$
\left\langle\Phi_{\mathrm{PB}}\right\rangle=\int d \phi \mathcal{P}_{2 \pi}(\phi) \phi
$$

where $\mathcal{P}_{2 \pi}$ is the Pegg-Barnett phase probability distribution cast to the $2 \pi$ interval. In a similar way, the mean value of the cosine of the phase difference is

$$
\left\langle\cos \Phi_{\mathrm{PB}}\right\rangle=\int d \phi \mathcal{P}_{2 \pi}(\phi) \cos \phi
$$

Finally, note that for the exponential of the phase difference (and similarly for the cosine and sine operators) the Pegg-Barnett approach gives just the SusskindGlogower phase-difference operator $E_{+} E_{-}^{\dagger}$, where $E_{+}$ and $E_{-}$are the Susskind-Glogower operators for each mode.

\section{DISCUSSION}

Before making any comparison among the evolutions of the phase difference according to the approaches discussed earlier, we want to pay special attention to the results of the casting procedure in the Pegg-Barnett formalism.

Of course it has no consequences when evaluating a periodic function of the phase difference, but for the phase difference itself and its variance the situation is quite different. We must stress that, after the restriction to a $2 \pi$ range, the mean value of the phase difference is not the difference of the mean values of the individual phases. Also, its variance cannot be obtained in terms of the variances and correlation of the one-mode phases. All this occurs due to the special character of this variable, and we can note that after the casting procedure the mean value and variance of the phase difference in the PeggBarnett formalism are closer to the ones obtained from the operator (3.22).

As a clear and important example of this discussion, we can consider the situation when one of the modes is in a state of random phase (for example, $\left|\psi_{+}, n_{-}\right\rangle$where $\psi_{+}$is arbitrary). It can be seen that its phase-difference probability distribution is uniform (because if one of the phases is random it seems that its difference with another one must also be random no matter how much the second one fluctuates) and time independent when the evolution is given by the Hamiltonian (2.11). So the mean value of any function of the phase difference is constant all through the propagation in the Kerr medium. However, each phase evolves differently when it is considered individually according to the Pegg-Barnett approach. In Fig. 1 we have represented this situation when the initial state is a coherent one with $\left|\alpha_{+}\right|^{2}=1$ in mode + and the vacuum in mode - . We can see that the expected behavior is satisfied by the two operators while the Stokes parameters predict a very different time variation.

The main differences between the Pegg-Barnett approach and the operator (3.22) are due to the discrete character of the possible phase values for the latter. Let us compare the mean values

$$
\begin{aligned}
\left\langle\Phi_{\mathrm{PB}}\right\rangle= & \left\langle\sum_{n=0}^{\infty} \sum_{n_{+}, n_{+}^{\prime}=0}^{n} \frac{1}{2 \pi} \int_{-\pi}^{\pi} d \phi \phi e^{i \phi\left(n_{+}^{\prime}-n_{+}\right)}\right. \\
& \times\left|n_{+}^{\prime}, n-n_{+}^{\prime}\right\rangle\left\langle n_{+}, n-n_{+} \mid\right\rangle \\
\langle\Phi\rangle= & \left\langle\sum_{n=0}^{\infty} \sum_{n_{+}, n_{+}^{\prime}=0}^{n} \sum_{r=0}^{n} \frac{1}{n+1} \phi_{r}^{(n)} e^{i \phi_{r}^{(n)}\left(n_{+}^{\prime}-n_{+}\right)}\right. \\
& \times\left|n_{+}^{\prime}, n-n_{+}^{\prime}\right\rangle\left\langle n_{+}, n-n_{+} \mid\right\rangle
\end{aligned}
$$

and similarly for the powers of the exponential of the phase. We can note their similar structure, and we can expect that both formalisms will get coincident results when the $r$ sum can be properly approximated by the 


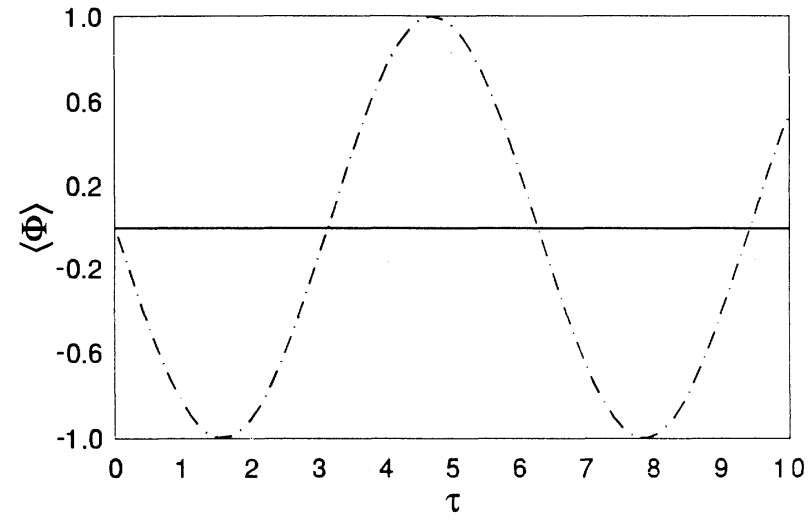

FIG. 1. Evolution of the mean phase difference for a coherent state with $\left|\alpha_{+}\right|^{2}=1$ and $\left|\alpha_{-}\right|^{2}=0$. The solid line represents the identical predictions of the phase-difference operator (3.22) and of the Pegg-Barnett approach, while the broken line is the values obtained from the Stokes parameters.

integral. This is tantamount to neglecting the discrete character and assuming a continuous one. A requirement to justify this replacement is that the mean values must involve only high $n$ terms in the summations; that is, when we are in the regime of high total photon numbers. This does not mean that both modes are highly excited, because this condition can be reached when only one of the modes is intense. On the other hand, we should expect differences in the regime where the discrete character becomes noticeable; that is, in the limit of small total photon numbers. As long as the total photon number is conserved by the evolution operator, if one of these conditions is satisfied initially, it is also satisfied all through the propagation in the Kerr medium.

According to this, we can expect similar results from both approaches in the limit of high photon numbers, as is shown in Fig. 2, where the initial state is a coherent one with $\left|\alpha_{+}\right|^{2}=6$ and $\left|\alpha_{-}\right|^{2}=1$. It can also be seen how the mean values and variances reflect the randomization of the phase difference. It can be checked that appreciable oscillations in the variances occur when we are in conditions to observe the appearance of macroscopically distinguishable states or Schrödinger cat states $[6,7]$.

This discussion concerning their similarity in this limit can also be done in terms of the phase-difference probability distributions

$$
\mathcal{P}_{2 \pi}(\phi)=\sum_{n=0}^{\infty}\left|\left\langle\phi^{(n)} \mid \psi\right\rangle\right|^{2},
$$

for the Pegg-Barnett approach, where

$$
\left|\phi^{(n)}\right\rangle=\frac{1}{\sqrt{2 \pi}} \sum_{n_{+}=0}^{n} e^{i \phi n_{+}}\left|n_{+}, n-n_{+}\right\rangle
$$

for any $\phi$, and

$$
P(\phi)=\left\{\begin{array}{cc}
\sum_{n, r}\left|\left\langle\phi_{r}^{(n)} \mid \psi\right\rangle\right|^{2} & \text { if } \phi=\phi_{r}^{(n)} \\
0 & \text { otherwise }
\end{array}\right.
$$

(a)

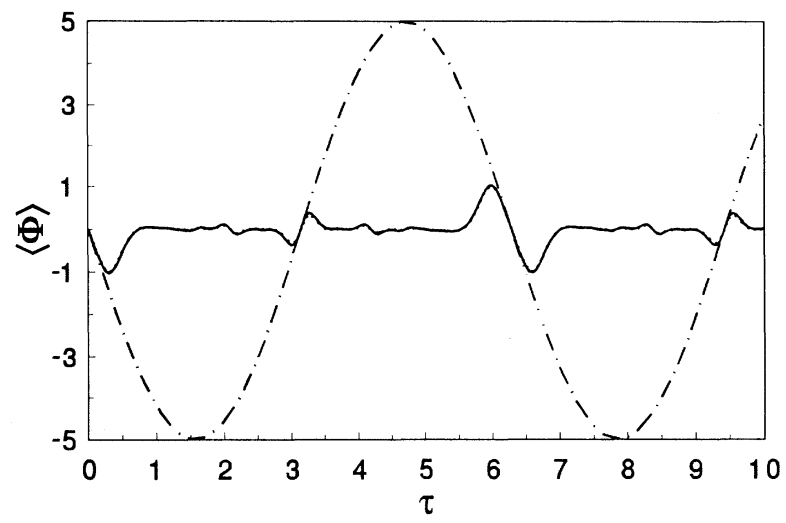

(b)

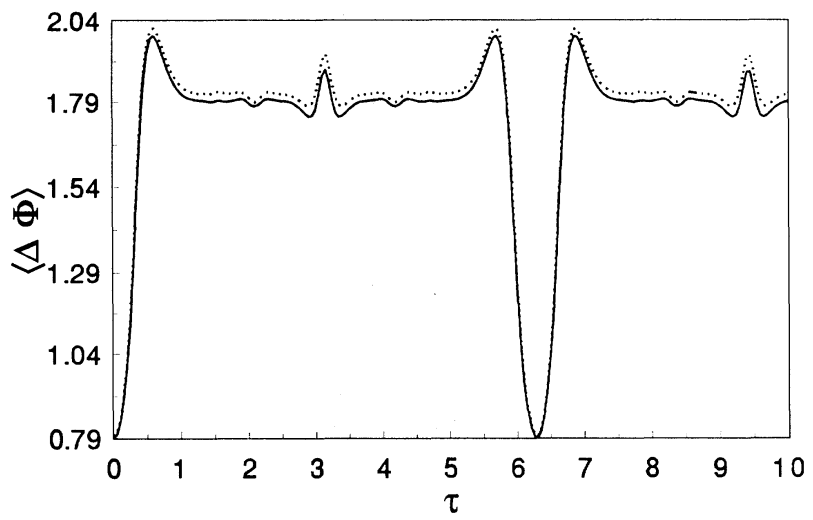

FIG. 2. (a) Evolution of the mean phase difference for a coherent state with $\left|\alpha_{+}\right|^{2}=6$ and $\left|\alpha_{-}\right|^{2}=1$. The lines have the same meaning as in Fig. 1. (b) Evolution of the variances for the phase-difference operator (solid) and the Pegg-Barnett phase difference (dotted).

for the phase operator (3.22), where in the sum $n$ and $r$ run only over those values for which

$$
\phi=\phi_{r}^{(n)}=\phi_{0}^{(n)}+\frac{2 \pi r}{n+1} .
$$

We can see again that their structure is quite similar, but while the first probability distribution is a function of the continuous variable $\phi$, the second one can only take values different from zero over a dense numerable set. So their form should look different, as is shown in Fig. 3, and this occurs in this case despite the fact that we are in the same limit of high photon numbers.

However, their common structure and the agreement of mean values just shown tell us that somehow they must be very close in this limit. To see more clearly how they can lead to the same results we can look at the $\phi$ axis with a finite resolution, dividing the whole range into intervals of the same length, and adding the points falling in the same (small but finite) interval. This smoothing procedure is actually done when we take the mean value 
(a)

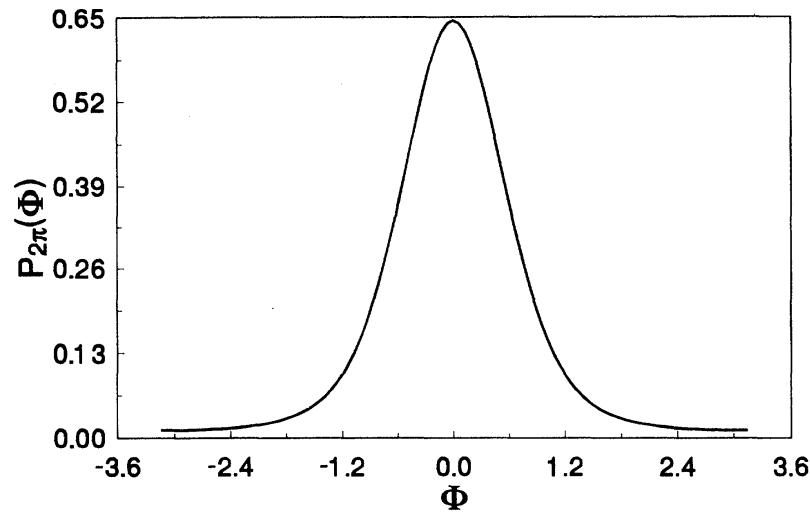

(b)

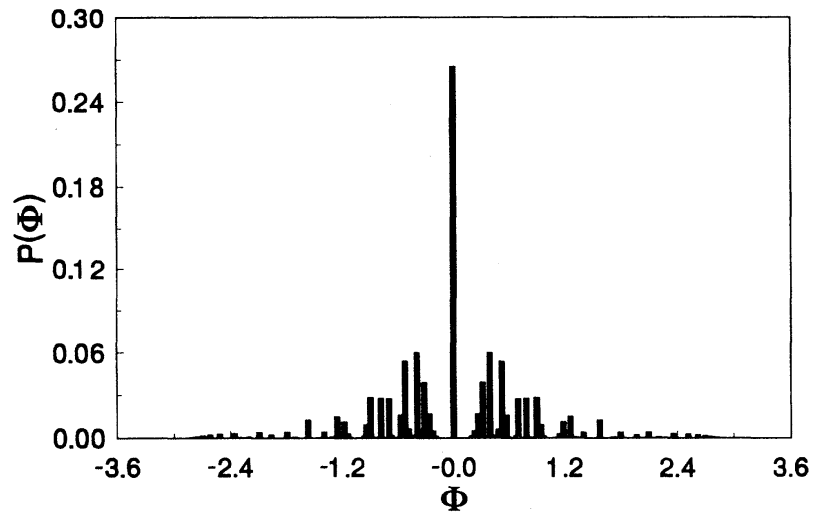

FIG. 3. Phase-difference probability distribution functions in $\tau=0$ for a coherent state with $\left|\alpha_{+}\right|^{2}=6$ and $\left|\alpha_{-}\right|^{2}=1$. (a) Probability distribution cast to the interval $2 \pi$ for the Pegg-Barnett phase difference. (b) Discrete probability distribution for the phase-difference operator (3.22).

of a slowly varying function of the phase difference. It also could be considered as the effect of the always finite resolution of a measuring device. After this handling we get some effective phase distribution in Fig. 4 (in which we have divided the whole range into 14 intervals) that looks closer to the Pegg-Barnett distribution. For higher photon numbers the length of the intervals needed to get a smoothed phase distribution closer to the Pegg-Barnett one is correspondingly less.

In the other limit of small total photon number it is clear that the discrete character will establish the differences between the two approaches. In Fig. 5 we have plotted the time evolution when the initial state is coherent with $\left|\alpha_{+}\right|^{2}=1$ and $\left|\alpha_{-}\right|^{2}=0.25$. We can see that, although they have different values, both curves follow the same qualitative behavior, despite the fact that they should have different properties in this regime.

It is easy to see that

$$
E=E_{+} E_{-}^{+}+\sum_{n} e^{i(n+1) \phi_{0}^{(n)}}|n, 0\rangle\langle 0, n|
$$

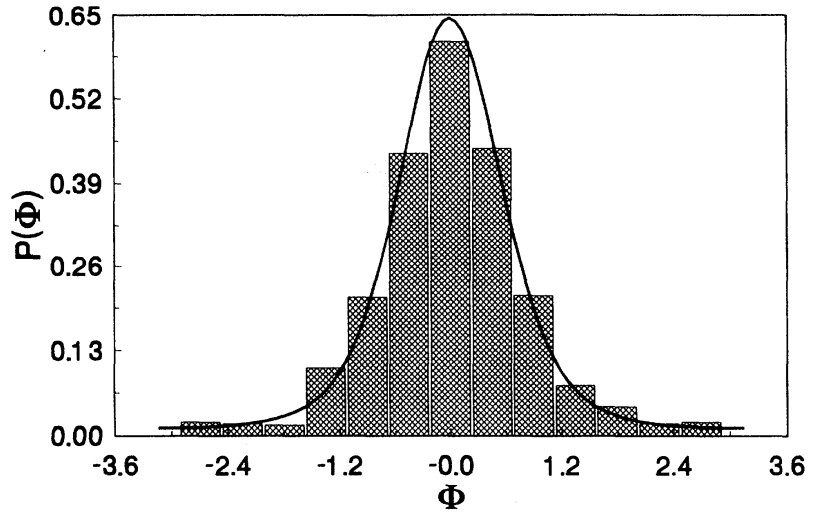

FIG. 4. Smoothed phase-difference distribution obtained from Fig. 3(b) by adding the points falling in intervals of length $\pi / 7$ and compared with the Pegg-Barnett distribution for the same state as in Fig. 3.

where $E_{+} E_{-}^{+}$is the Susskind-Glogower phase difference for both modes, which coincides with the result obtained in the Pegg-Barnett approach. Furthermore, since the mean value of the extra term

$$
\sum_{n} e^{i(n+1) \phi_{0}^{(n)}}|n, 0\rangle\langle 0, n|
$$

(a)

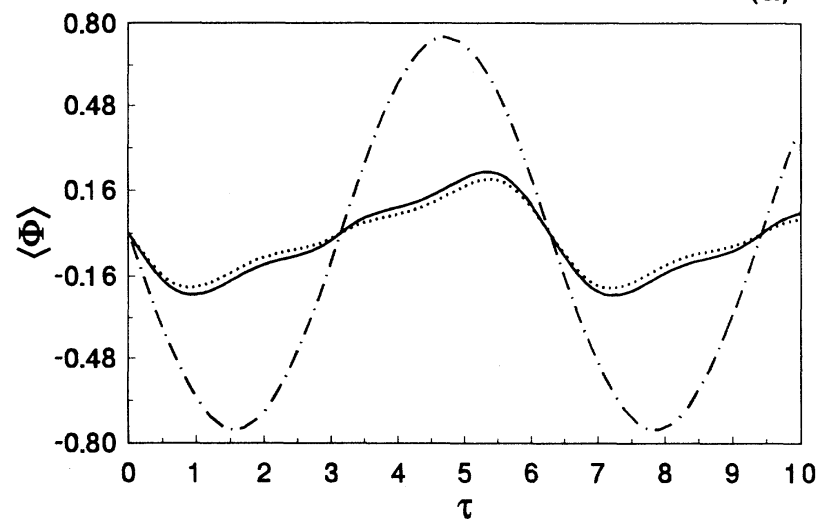

(b)

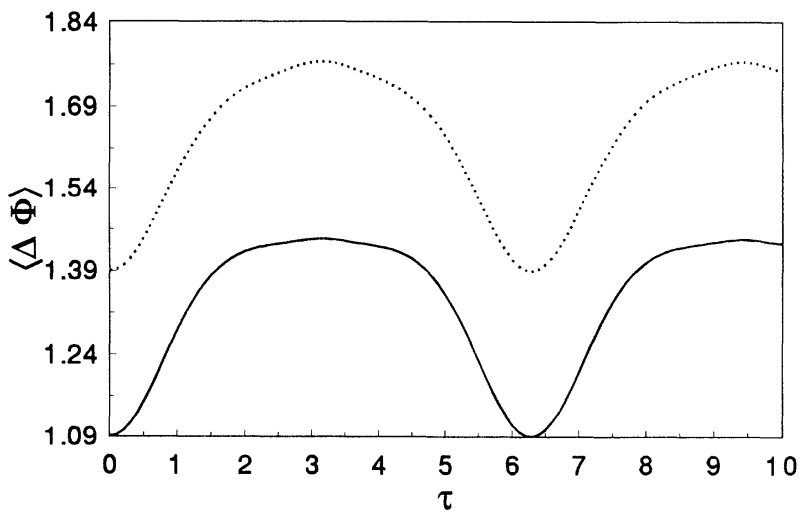

FIG. 5. The same as in Fig. 2 but now in the quantum regime of small photon numbers: $\left|\alpha_{+}\right|^{2}=1$ and $\left|\alpha_{-}\right|^{2}=0.25$. 
is constant in the evolution, the differences between the exponential of the phase difference (and, in particular, the cosine) reduce to a constant for both approaches (as is clearly seen in Fig. 6). This constant tends to zero in the limit of high photon numbers.

It is worth mentioning that, as seen in the figures, the phase fluctuations calculated by means of the PeggBarnett approach are larger than those obtained by the phase-difference operator. Since in general a coarsegraining procedure enhances fluctuations, this is consistent with the fact that, after smoothing, the phase distribution by the phase-difference operator method approaches the Pegg-Barnett phase distribution function.

We should note as well the periodic character of the evolution of the phase difference. This is a common feature of the three approaches discussed. For the Stokes operators we can see that

$$
\begin{aligned}
U^{\dagger}(\tau) a_{+}^{\dagger} a_{-} U(\tau)= & a_{+}^{\dagger} \exp \left[i \tau\left(\kappa_{2}-\kappa_{1}\right)\right. \\
& \left.\times\left(a_{+}^{\dagger} a_{+}-a_{-}^{\dagger} a_{-}\right)\right] a_{-},
\end{aligned}
$$

and therefore the Stokes parameters are $2 \pi$ periodic on $\tau\left(\kappa_{2}-\kappa_{1}\right)$ due to the integer nature of the spectrum of $a_{+}^{\dagger} a_{+}$and $a_{-}^{\dagger} a_{-}$in the quantum case.

To show this behavior in the Pegg-Barnett formalism and on the operator (3.22), we can make use of their similar structure for the probability distribution function. In both cases, they are obtained from the projection operators $\left|\phi^{(n)}\right\rangle\left\langle\phi^{(n)}\right|$, where

$$
\left|\phi^{(n)}\right\rangle=\frac{1}{\sqrt{2 \pi}} \sum_{n_{+}=0}^{n} e^{i \phi n_{+}}\left|n_{+}, n-n_{+}\right\rangle .
$$

In the Pegg-Barnett formalism $\phi^{(n)}$ can take any value, while in the other case $\phi^{(n)}$ takes only $n+1$ values for each $n$. The evolution of this projection operator is (a)

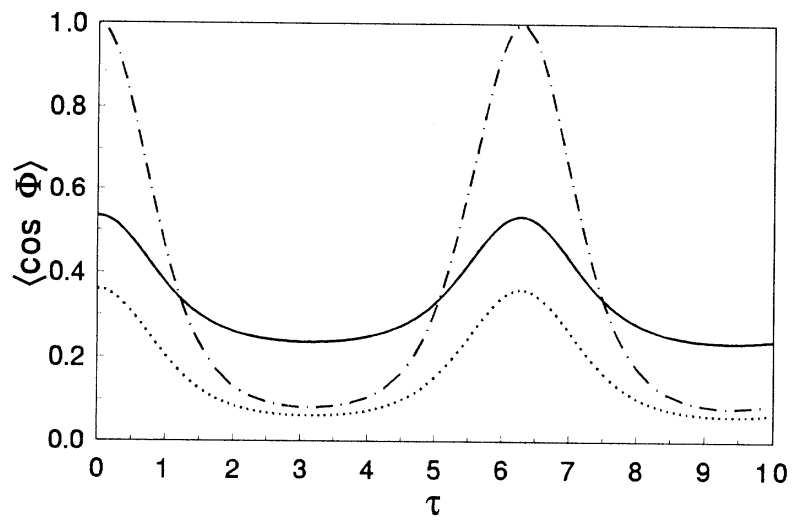

(b)

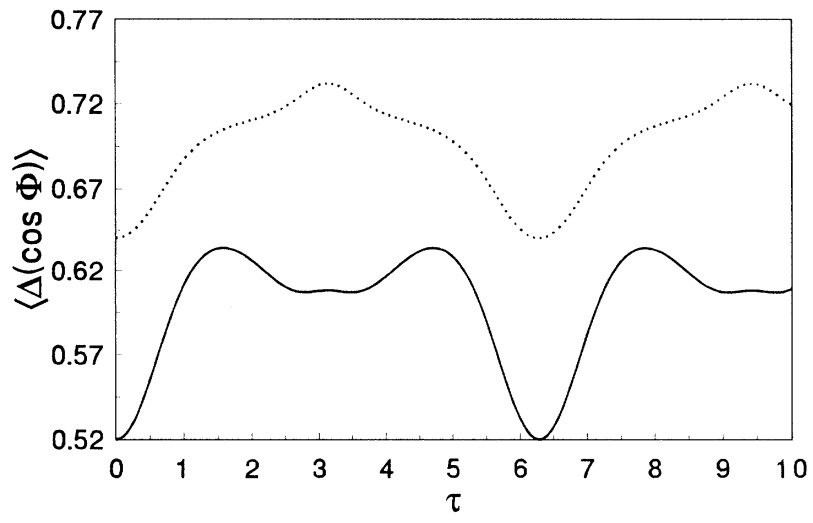

FIG. 6. (a) Mean values of the cosine of the phase difference for a coherent state with $\left|\alpha_{+}\right|^{2}=1$ and $\left|\alpha_{-}\right|^{2}=0.25$. (b) Variances of the cosine of the phase difference.

$$
\begin{aligned}
U^{\dagger}(\tau)\left|\phi^{(n)}\right\rangle\left\langle\phi^{(n)}\right| U(\tau) & =\frac{1}{2 \pi} \sum_{n_{+}, n_{+}^{\prime}=0}^{n} U^{\dagger}(\tau)\left|n_{+}, n-n_{+}\right\rangle e^{i\left(n_{+}-n_{+}^{\prime}\right) \phi}\left\langle n_{+}^{\prime}, n-n_{+}^{\prime}\right| U(\tau) \\
& =\frac{1}{2 \pi} \sum_{n_{+}, n_{+}^{\prime}=0}^{n} e^{i\left(n_{+}-n_{+}^{\prime}\right) \phi} e^{i \tau\left(\kappa_{2}-\kappa_{1}\right)\left[n_{+}\left(n-n_{+}\right)-n_{+}^{\prime}\left(n-n_{+}^{\prime}\right)\right]}\left|n_{+}, n-n_{+}\right\rangle\left\langle n_{+}^{\prime}, n-n_{+}^{\prime}\right|
\end{aligned}
$$

It is evident that the evolution is $2 \pi$ periodic in $\tau\left(\kappa_{2}-\kappa_{1}\right)$, and so will be the corresponding probability distribution function, regardless of the discrete or continuous character of the $\phi$ variable.

It should be pointed out that this periodicity in the evolution of the phase difference is present regardless of the particular values of $\kappa_{2}$ and $\kappa_{1}$, because they always appear in the form $\kappa_{2}-\kappa_{1}$. In particular, in all the figures we have taken $\kappa_{2}-\kappa_{1}=1$. Contrary to the classical solution (2.9), this periodicity does not depend on the intensity of the fields, because its origin lies in the discrete nature of the corresponding operators.

Equivalently, the Hamiltonian (2.11) can be rearranged as

$$
\begin{aligned}
H= & \frac{\hbar}{2}\left\{\kappa_{1}\left[\left(n_{+}+n_{-}\right)^{2}-\left(n_{+}+n_{-}\right)\right]\right. \\
& \left.+2\left(\kappa_{2}-\kappa_{1}\right) n_{+} n_{-}\right\}
\end{aligned}
$$

Since the phase difference commutes with the total number, the relevant part for the evolution is given by the last term, which depends just on the difference between $\kappa_{2}$ and $\kappa_{1}$. Thus the evolution will be periodic due to the integer character of $n_{+}$and $n_{-}$for any value of $\kappa_{2}$ and $\kappa_{1}$. When $\kappa_{2}=\kappa_{1}$ there is no evolution at all for the phase difference.

In summary, what we expect to have accomplished is a complete quantum description of the phase properties of light propagating in a Kerr medium. Starting from the 
Stokes operators, we have extracted, via a polar decomposition, a unitary phase-difference operator allowing a clear description of the polarization state of the field. These predictions have been compared with those based on the Pegg-Barnett approach. Although for large numbers of photons both operators give equivalent results (the agreement is fairly good even for $n \gtrsim 4$ ), quite different from the classical Stokes parameters, in the regime of small photon numbers some subtle differences appear, associated with the discrete eigenvalue spectrum of the phase-difference operator.

\section{ACKNOWLEDGMENTS}

Two of us (A.L. and L.L.S.-S.) wish to thank the Institute of Physics of the Adam Mickiewicz University for kind hospitality. Helpful discussions with Dr. A. Miranowicz are also acknowledged.

\section{APPENDIX: CASTING PROCEDURE FOR THE PEGG-BARNETT PHASE-DIFFERENCE DISTRIBUTION}

The Pegg-Barnett formalism for the phase properties of a two-mode field is constructed from the individual phases corresponding to each mode. The procedure can be summarized by the joint probability distribution function obtained in terms of the Susskind-Glogower phase states

$$
\left|\phi_{+}, \phi_{-}\right\rangle=\frac{1}{2 \pi} \sum_{n_{+}, n_{-}=0}^{\infty} e^{i\left(n_{+} \phi_{+}+n_{-} \phi_{-}\right)}\left|n_{+}, n_{-}\right\rangle
$$

as

$$
P_{\psi}\left(\phi_{+}, \phi_{-}\right)=\left|\left\langle\phi_{+}, \phi_{-} \mid \psi\right\rangle\right|^{2}
$$

In our case we are interested not in the phase of each mode but in the phase difference, which is the relevant variable in a lot of two-mode problems. So we can ask for the probability distribution function corresponding to the phase-sum and phase-difference variables

$$
\begin{aligned}
& \theta=\phi_{+}+\phi_{-}, \\
& \phi=\phi_{+}-\phi_{-},
\end{aligned}
$$

that is,

$$
\begin{aligned}
& \phi_{+}=\frac{\theta+\phi}{2}, \\
& \phi_{-}=\frac{\theta-\phi}{2} .
\end{aligned}
$$

However, due to the $2 \pi$-periodic character of all these variables, the probability distribution corresponding to $(\theta, \phi)$ cannot be obtained simply by putting (A4) into (A2). Note that in (A3) it seems that the range of $(\theta, \phi)$ should be a $4 \pi$ interval, or, equivalently, that (A4) is not compatible with the $2 \pi$ periodicity. Then the substitution of (A4) into (A2) must be followed by a procedure casting $\theta$ and $\phi$ into $2 \pi$ ranges. To do so one has to deal very carefully with the regions for all these phases. The reason is that the same $\bmod (2 \pi)$ value for $(\theta, \phi)$ is obtained from two different values of $\left(\phi_{+}, \phi_{-}\right)$; specifically, we will get the same $(\theta, \phi)$ from $\left(\phi_{+}, \phi_{-}\right)$and $\left(\phi_{+}+\pi, \phi_{-}+\pi\right)$ [this last pair must be understood as $\bmod (2 \pi)]$. So to obtain the probability for the $(\theta, \phi)$ values we must add the probabilities corresponding to individual phases $\left(\phi_{+}, \phi_{-}\right)$and $\left(\phi_{+}+\pi, \phi_{-}+\pi\right)$ in such a way that [taking into account the Jacobian of transformation (A4)]

$$
\begin{aligned}
\mathcal{P}_{2 \pi}(\theta, \phi)= & \frac{1}{2}\left[P_{\psi}\left(\frac{\theta+\phi}{2}, \frac{\theta-\phi}{2}\right)\right. \\
& \left.+P_{\psi}\left(\frac{\theta+\phi}{2}+\pi, \frac{\theta-\phi}{2}+\pi\right)\right],
\end{aligned}
$$

$\mathcal{P}_{2 \pi}(\theta, \phi)$ being now $2 \pi$ periodic over the $\theta$ and $\phi$ variables.

In this work, we are interested in the marginal distribution for the phase difference, which can be obtained just by integrating (A5) over the phase-sum variable $\theta$. This probability distribution can also be obtained in a very simple way just by noting that it must give the same mean value of any periodic function of the phase difference as the joint probability distribution (A2) (for an alternative and careful derivation, see the original work of Barnett and Pegg [24]).

Then we can impose the requirement

$$
\int d \phi e^{i k \phi} \mathcal{P}_{2 \pi}(\phi)=\int d \phi_{+} d \phi_{-} e^{i k\left(\phi_{+}-\phi_{-}\right)} P_{\psi}\left(\phi_{+}, \phi_{-}\right)
$$

for any integer $k . \mathcal{P}_{2 \pi}(\phi)$ is then completely fixed by this requirement as well as from the $\theta$ integration of (A5), and can be expressed as

$$
\mathcal{P}_{2 \pi}(\phi)=\int d \theta|\langle\phi+\theta, \theta \mid \psi\rangle|^{2}=\sum_{n=0}^{\infty}\left|\left\langle\phi^{(n)} \mid \psi\right\rangle\right|^{2},
$$

where $\left|\phi^{(n)}\right\rangle$ is the same as (4.3), as can be shown by direct computation.
[1] Y. R. Shen, The Principles of Nonlinear Optics (Wiley, New York, 1985).

[2] H. H. Ritze and A. Bandilla, Opt. Commun. 29, 126 (1979).

[3] R. Tanaś and S. Kielich, Opt. Commun. 30, 443 (1979).

[4] R. Tanaś and S. Kielich, Opt. Commun. 45, 351 (1983); Opt. Acta 31, 81 (1984).
[5] G. J. Milburn and C. A. Holmes, Phys. Rev. Lett. 56, 2237 (1986).

[6] Ts. Gantsog and R. Tanaś, Quantum Opt. 3, 33 (1991).

[7] K. Tara, G. S. Agarwal, and S. Chaturvedi, Phys. Rev. A 47, 5024 (1993).

[8] Y. Yamamoto, S. Machida, N. Imoto, M. Kitagawa, and G. Björk, J. Opt. Soc. Am. A 4, 1645 (1987). 
[9] G. J. Milburn, Phys. Rev. A 33, 674 (1986).

[10] M. Kitagawa and Y. Yamamoto, Phys. Rev. A 34, 3974 (1986).

[11] G. S. Agarwal and R. R. Puri, Phys. Rev. A 39, 2969 (1989).

[12] R. Tanaś, in Coherence and Quantum Optics, edited by L. Mandel and E. Wolf (Plenum, New York, 1984), p. 645.

[13] P. Carruthers and M. M. Nieto, Rev. Mod. Phys. 40, 441 (1968).

[14] J. Bergou and B. G. Englert, Ann. Phys. (N.Y.) 209, 479 (1991).

[15] S. M. Barnett and D. T. Pegg, J. Phys. A 19, 3849 (1986).

[16] A. Luis and L. L. Sánchez-Soto, Phys. Rev. A 48, 4702 (1993).
[17] L. L. Sánchez-Soto and A. Luis, Opt. Commun. 105, 84 (1994).

[18] D. T. Pegg and S. M. Barnett, Europhys. Lett. 6, 483 (1988); J. Mod. Opt. 36, 7 (1989).

[19] Ts. Gantsog and R. Tanaś, J. Mod. Opt. 38, 1537 (1991).

[20] G. S. Agarwal and R. R. Puri, Phys. Rev. A 40, 5179 (1989).

[21] M. Born and E. Wolf, Principles of Optics (Pergamon, Oxford, 1980).

[22] J. M. Jauch and F. Rohrlich, The Theory of Photons and Electrons (Addison-Wesley, Reading, 1959).

[23] R. Tanaś and Ts. Gantsog, Opt. Commun. 87, 369 (1992).

[24] S. M. Barnett and D. T. Pegg, Phys. Rev. A 42, 6713 (1990). 